\title{
Assessment of aesthetic and functional improvement of patients with mid-facial hypoplasia corrected using mid maxillary osteodistraction (MMOD) with a tooth-borne distractor
}

\author{
Dias DK, Fernando PDC \\ Oral \& Maxillo-Facial Surgery Unit, Teaching Hospital, Karapitiya, Sri Lanka. \\ Correspondence: Dr. PDC Fernando \\ e-mail: dilan4fernando@gmail.com
}

\begin{abstract}
Introduction: A method of mid maxillary distraction osteogenesis using tooth borne custom-made device is being performed on cleft patients in Oral and Maxillofacial unit, Teaching Hospital, Karapitiya, Sri Lanka, over the past ten years. The technique is simple, inexpensive and less time consuming. Aims of this study were to assess the level of improvement of maxillary unit length, facial appearance and quality of speech of patients following correction of mid facial hypoplasia using MMOD.

Methods: Patients with midfacial hypoplasia ( $\mathrm{n}: 10, \mathrm{M}: \mathrm{F}-1: 1$, Age range : 14-22 yrs) surgically corrected with MMOD technique during 2013 were included. Increase of maxillary unit length, SNA \& ANB angles and over-jet were assessed with study-models and cephalometric analysis pre-surgically and one year after surgery. The quality of speech was assessed using resonance, nasal air emission and consonant production error pre and post surgically.

Results: All patients showed positive over-jet increase (mean: $4.3 \mathrm{~mm}$ ) and improved anterior-posterior dimensions of maxilla (SNA mean increase: $8.5^{\circ}$, ANB mean increase: $8.8^{\circ}$ and average increase of Maxillary Unit Length/ANS - PNS: $6.2 \mathrm{~mm})$. Findings were statistically significant $(\mathrm{p}<0.05)$. All patients accepted their current facial appearance. $60 \%$ of patients showed reduced hyper-nasality and nasal air emission in speech.

Conclusions: MMOD is a less expensive, less invasive orthognathic surgical procedure where adequate distraction is possible with minimum relapse. Bone formation on either side of osteotomy cuts expand soft tissues as well, to improve facial aesthetics and reduce velo-pharyngeal insufficiency to improve speech.
\end{abstract}

Key words: Mid-maxillary distraction osteogenesis, tooth- borne appliance.

\section{Introduction}

Congenital oro-facial clefts and related growth defects are prime causes of maxillary/ mid facial hypoplasia (1). Repaired cleft lip and palate itself have a negative impact on mid-facial growth. Sixty percent of children with surgically corrected cleft lip and palate will require correction of maxillary hypoplasia and $25 \%$ of them are beyond the boundaries of conventional orthodontic treatments (1). Class III skeletal base, crowded upper-arch with insufficient alveolar space and clefts causing malocclusion, facial profile defects in all three planes(mainly due to mid facial retrusion) and altered soft tissue harmony in nose, orbit and upper lip have negative functional and aesthetic/social impact on these patients. Under-developed and insufficient length of soft palate causes speech and swallowing problems due to velo-pharyngeal insufficiency (VPI). Moderate maxillary hypoplasia usually associated with oro-facial clefts can be managed with mid maxillary osteo-distraction (MMOD) technique. 
Distraction osteogenesis (DO) is the surgical technique in which new bone formation is induced by gradual separation of bony segments after a precise osteotomy. The method produces unlimited quantity of living bone directly by controlled mechanical distraction of the bony segments. The concept of bone lengthening by distraction was described by Codivilla in 1905 and proven by Ilizarov a Russian surgeon in 1951 by applying it to lengthen limbs (2). The use of DO on craniofacial skeleton was introduced by McCarthy in 1992 to correct a congenital hypoplastic mandible (3).

DO comprise of four basic steps as osteotomy phase, latency phase, distraction phase and consolidation phase. Gradual expansion of surrounding soft tissues, muscles and mucosa adapt to the bony changes causing increase in the size of soft tissue envelope. This is known as distraction histogenesis which enables minimum relapse comparing to advancement osteotomy procedures $(4,5)$.

Le Fort I level osteo-distraction was initially used for correction of moderate maxillary hypoplasia in cleft patients (6), but jutting out activation devices on maxillary vestibules cause continuous intra-oral trauma and not aesthetically pleasing to wear for a long time.

Anterior-posterior unidirectional tooth-borne intraoral distraction device (1) was invented to over-come the situation. This technique is being practiced at Oral and Maxillofacial Unit (OMFU), Teaching Hospital Karapitiya, Sri Lanka since 2002; at present about 15-20 patients per year are treated using MMOD for correction of mid-facial hypoplasia. It is a definitive surgery mostly performed at the age of growth completion to gain aesthetic and functional improvement on patients who underwent initial surgical corrections for mid facial defects (cleft palate with or without cleft lip).

Aim of this study was to emphasize the importance and validity of this less invasive and inexpensive specific surgical procedure, which is a favorable option with utilization of available facilities in Sri Lanka.

\section{Methods}

Patients with mid-facial hypoplasia secondary to repair of cleft palate with or without cleft lip (n-10, $\mathrm{M}: \mathrm{F}-1: 1$, Age range : 14 - 22 yrs) surgically corrected with MMOD technique during 2013 were included in this series. Over-jet and increase of maxillary unit length [distance between anterior nasal spine (ANS) to posterior nasal spine (PNS)] assessed with cephalometric analysis and studymodels pre-surgically and one year after surgery. The quality of speech was assessed using resonance, nasal air emission and consonant production error pre and one year post surgically by a speech and language therapist. Patients' appearance and acceptance were assessed by a short interview.

\section{Surgical procedure}

All patients had undergone pre-operative orthodontic treatments to create surgical space in the maxillary arch for osteotomy cuts. Nickel-Titanium coil springs were used to achieve the desired root separation for osteotomy cuts preferably between the maxillary second premolar and first molar teeth. Where this was not achievable, a space created between the first and second maxillary molar teeth became the alternative choice (1).

Custom-made tooth-borne distractor was constructed with orthodontic guidance on study model (Figure: 1). Molar and premolar bands were soldered to orthodontic hyrax screw for construction of the distraction device. The screw was aligned in the sagittal plane to achieve an antero-posterior vector with slight inclination anteriorly.

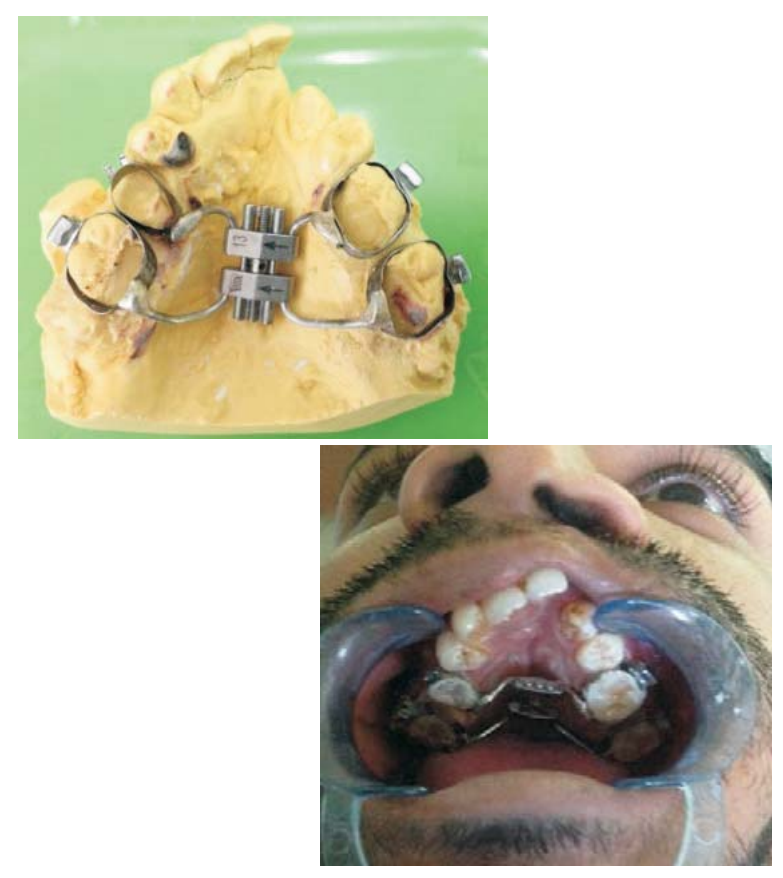

Figure 1: Distraction device on model and in situ 
Osteotomy phase was performed under general anesthesia. Anterior le-fort I level osteotomy combined with transverse mid-maxillary osteotomy cut through orthodontically created spaces of upper arch was done (Figure 2).
Minimum periosteal striping and preservation of palatal muco-periosteum is highly desirable to nourish separated anterior maxillary segment. Maximum amount of maxilla was incorporated to osteotomized segment.
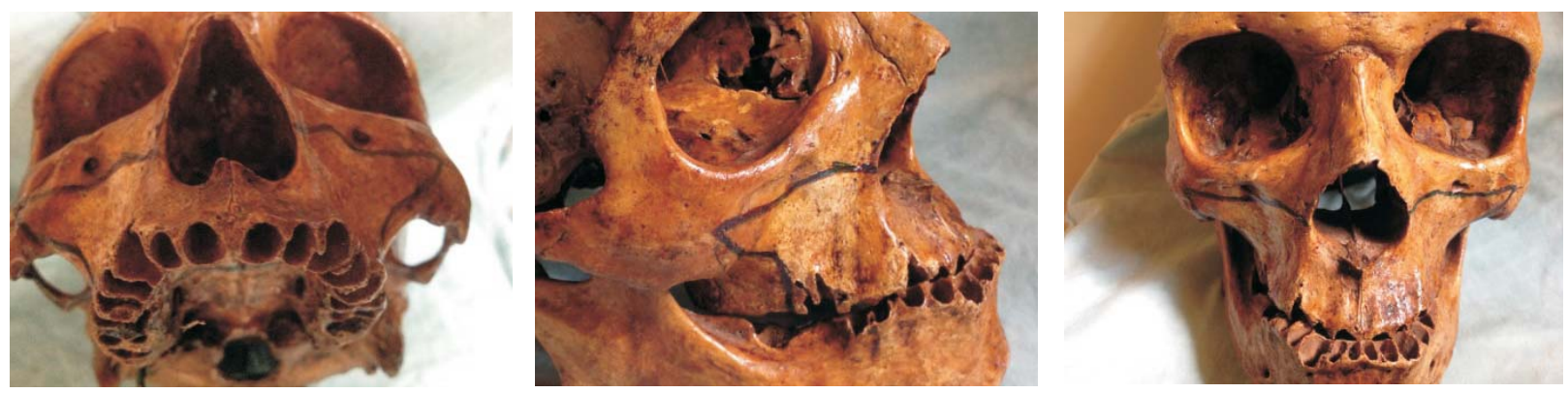

Figure 2: Osteotomy cuts marked on skull

Distraction was delayed for 5-7 days (Latency phase) until soft callus formation to bridge the bony segments after the bone healing was commenced. Intra-oral distracter was cemented to preplanned teeth.

During Distraction phase, intra-oral distracter was activated $1 \mathrm{~mm}$ per day (two turns in the morning and two in the evening). One turn opens $0.25 \mathrm{~mm}$ of the device. Maximum distraction of $13 \mathrm{~mm}$ (vary with the type of screw) was achieved by two weeks.

Consolidation phase is theoretically twice as distraction phase (four weeks). The device was kept in situ for three months after maximum activation until callus get matured and remodeled to minimize relapsing.

\section{Results}

Improvement of maxillary deficiency was assessed using following cephalometric measurements, pre surgically and after one year post surgically.

All patients showed increase of maxillary unit length (distance between ANS to PNS) with average increase of $6.2 \mathrm{~mm}$. All the study subjects showed increase of SNA (Sella-Nasion-A point Angle) and ANB (A point-Nasion-B point Angle) angles with the mean increase of $8.5^{\circ}$ and $8.8^{\circ}$, which improved the anterior-posterior dimensions of maxilla. Mean increase of $4.3 \mathrm{~mm}$ was observed in positive over-jet increase in all patients. These findings were statistically significant $(\mathrm{P}<0.05)$ (Table 1$)$.

Table 1: Improvement of facial dimensions

\begin{tabular}{|lllc|}
\hline $\begin{array}{l}\text { Cephalometric } \\
\text { analysis }\end{array}$ & $\begin{array}{l}\text { Average } \\
\text { Increase }\end{array}$ & t value & p value \\
\hline $\begin{array}{l}\text { Maxillary Unit } \\
\text { Length }\end{array}$ & $6.2 \mathrm{~mm}$ & 9.7 & $<0.001$ \\
SNA angle & $8.5^{0}$ & 9.89 & 0.001 \\
ANB angle & $8.8^{0}$ & 9.99 & 0.001 \\
Over jet & $4.3 \mathrm{~mm}$ & & $<0.001$ \\
\hline
\end{tabular}

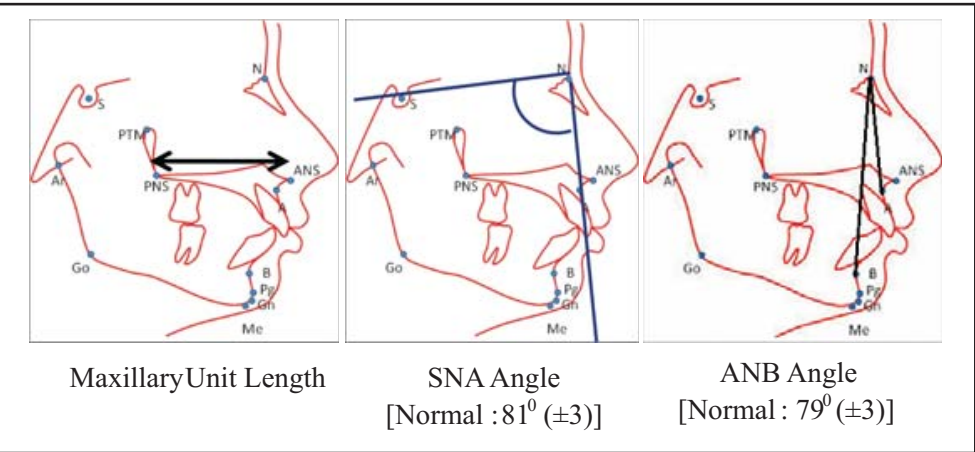


With the above positive changes, facial profile was changed from concave to convex, with correction of skeletal class III mal-occlusion to class II. Nasolabial angle was reduced. All patients accepted their current facial appearance than previous but preferred to have more nasal prominence (Figure 3).

According to the pre operative and one year post operative speech assessment, $60 \%$ of patients showed reduce hypernasality and nasal-emission after treatment with an improvement of VPI.

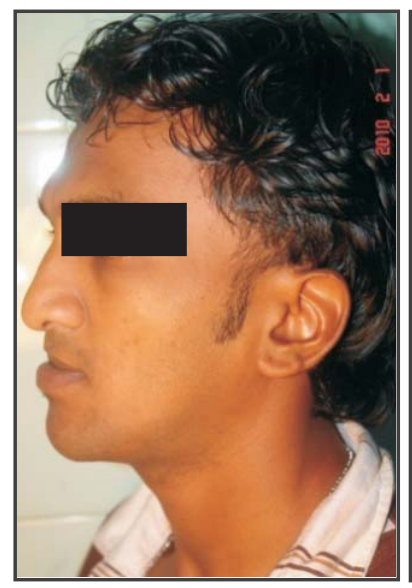

Pre-operative profile

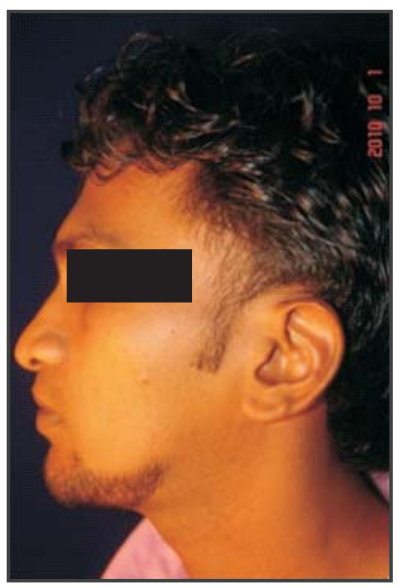

Post-operative profile
Figure 3: Improvement of facial appearance

\section{Discussion}

Hypoplastic maxilla due to oro-facial clefts results in functional problems as dento-alveolar discrepancy, insufficient space for teeth in alveolar process and clefts, speech and swallowing defects due to fistulae and VPI. Facial profile may alter in all three planes as facial soft tissue harmony of nose; orbit and upper lip are disturbed. Alteration of facial growth causes psychological and social impact.

The patient's main request in dento-facial deformity treatment is a new aesthetical balance of the face involving good occlusion, masticatory function, aesthetics of the smile and facial profile (7).

Among variety of treatment options for correction of maxillary hypoplasia of patients with oro-facial clefts, Le Fort I maxillary advancement osteotomy is widely used as it is accomplished of correcting maxillary hypoplasia in all three dimensions (6).
Depending on the severity of the discrepancy, the management protocol may vary but mostly performed in mild localized cases where as modified Le Fort III osteodistraction and Rigid External Devices (RED) are used in severe maxillary hypoplasia as in syndromic cases (8). Maxillary osteodistraction is often employed for correction of maxillary hypoplasia in patients with cleft lip and palate (9). The degree of correction and stability with DO is better than conventional osteotomies, rigid fixation, and bone grafting (10). Bone anchored internal distractors are more aesthetically acceptable and easy to wear but the main disadvantage is the need for a second surgery for removal at the end of treatment. Further, they have poor vector control and the distraction achievable is limited by the length of the screw incorporated in the device. Both, external and internal devices are expensive and need adequate bone above the root apices for safe placement to ensure good anchorage (1).

A relatively new approach for maxillary advancement by maxillary distraction using a combined surgical and modified orthodontic technique with tooth-borne custom-made osteodistractor (1) is a versatile treatment option on moderate mid facial hypoplasia patients with oro-facial clefts. Histogenesis is the main advantage of MMOD over Le Fort I maxillary advancement osteotomy as it creates bone as well as soft tissues that minimize relapses. The maxillary arch alveolar space created was effectively utilized for orthodontic correction of maxillary crowding. Since the posterior maxilla is not involved in surgical procedure, potential bleeding risk is minimum. Length of maxillary unit increase in anteriorposterior direction (ANS to PNS distance) improves VPI (II). Comparatively lesser reciprocal distraction of posterior maxillary segment improves the structural defect of the velopharyngeal sphincter by increasing the palatal length resulting in reduction of VPI. As a result there is a $60 \%$ reduction of hypernasality and nasal-emission in speech after treatment which would get worsened by le fort I advancement osteotomy. Adequate distraction is possible with multiple surgical attempts with provision of sufficient time intervals for bone remodeling and maturation. The method of DO can be used as an alternative to bone grafting and osteotomies. DO in contrast appear to provide low operative and post operative morbidity. 
With this technique tipping movements of premolars on which distractor arms were cemented anterior to osteotomy cut would occur and have to be corrected orthodontically. Further, any unfavorable fracture of maxilla posterior to osteotomy cut would not provide desired results of the technique. Defective distraction vectors and insufficient distraction are the drawbacks of the internal distractors (9).

According to our results, we were able to achieve significant improvements in facial appearance and speech in all patients. The appliance was well tolerated with high patient compliance. In conclusion this relatively simple and cost effective technique corrects mild to moderate maxillary hypoplasia, with the utilization of available facilities and is very much suited to third world and developed countries.

\section{References}

1. Dias DK, De Silva HL, Senadeera WM. Mid-maxillary distraction osteogenesis of cleft patients using a special custom-made tooth-borne device. Ceylon Medical Journal 2011; 56: 108-11.

2. Codvilla A. On the means of lengthening in the lower limbs, the muscles and tissues which are shortened through deformity. American Journal of Orthopaedic Surgery 1905; 2:353-8.

3. McCarthy JG, Schreiber J, Karp N, Thorne CH, Grayson BH. Lengthening the human mandible by gradual distraction. Plastic and Reconstructive Surgery1992; 89: $1-8$.
4. Booth PW. Orthognathic surgery. Maxillofacial surgery. Second edition. Missouri: Churchill Livingstone; 1999: 1155-289.

5. Proffit WR, White RP, Sarver DM. Contemporary treatment of dentofacial deformity. First edition. Mosby, 2003: 12: 357-364.

6. Drommer RB. The history of the LeFort I osteotomy. Journal of Maxillofacial Surgery1986; 14: 119-22.

7. Antonio Cortese. Lefort I osteotomy for maxillary repositioning and distraction techniques. www.intechopen. com

8. Polley JW, Figueroa AA. Rigid external distraction: its application in cleft maxillary deformities. Journal of Plastic Reconstructive Surgery 1998; 102(5): 1360-72.

9. Takigawa Y, Uematsu S, Takada K. Maxillary advancement using distraction osteogenesis with intraoral device: Angle Orthodontist 2010;80(6).

10. Chin M. The role of distraction osteogenesis in oral And Maxillofacial Surgery: Journal of Oral Maxillofacial Surgery 1998; 56: 805-6.

11. Janulewicz J, Costello BJ, Buckley MJ, Ford MD, Close J, Gassner R. The effect of Le Fort I osteotomies on velopharyngeal and speech functions in cleft patients. Journal of Oral Maxillofacial Surgery 2004; 62: 308-14. 